\title{
CT gently: a mobile tool for CT/CBCT dose and cancer risk estimation
}

\begin{abstract}
Computed tomography (CT) and cone-beam computed tomography (CBCT) scans have been widely used in the radiotherapy of cancers worldwide. Yet, so far there has been no efficient way available to estimate the organ dose and cancer risk originated from those imaging studies. To address this issue, we have recently developed an iPhone app called CT Gently that can be used to estimate organ doses and associated cancer risk from CT and CBCT scans based on individual anatomy and scan mode. In addition, the CT Gently app can be used to generate optimized $\mathrm{mAs}$ and $\mathrm{kVp}$ settings for personalized low-dose $\mathrm{CT}$ and CBCT scans.
\end{abstract}

Volume I Issue I - 2014

\author{
Jun Deng,' Huajun Zhang, ${ }^{2}$ Hai $\mathrm{Qi}^{2}$ \\ 'Department of Therapeutic Radiology, Yale University, USA \\ ${ }^{2}$ Komatsu Business Service Co., Chiyoda-ku, Japan
}

Correspondence: Jun Deng, Department of Therapeutic

Radiology of Yale University School of Medicine, Yale University, Yale-New Haven Hospital, New Haven, CT, USA,

Email jun.deng@yale.edu

Received: April 23, 2014 | Published: May 16, 2014

\section{Background}

In the past few decades, CT has been widely used in all kinds of diagnostic procedures and proved clinically effective. ${ }^{1}$ The very past decade has also seen the dramatic increase in the applications of $\mathrm{CBCT}$ in the radiotherapeutic management of cancers. ${ }^{2}$ Although implemented differently in the clinic, the radiation dose from both CT and CBCT scans has been a great concern among the clinicians and the general public, particularly to the children, as they are far more susceptible than the adults to radiation-induced late effects such as growth retardation and second malignancies. ${ }^{1,3}$

While CT Dose Index (CTDI) has been widely used in categorizing radiation doses from $\mathrm{CT}$ and $\mathrm{CBCT}$ scanners, it is a scanner-dependent index and not directly related to the organ dose in the patient being scanned. ${ }^{4}$ On the other hand, modern radiotherapy treatment planning systems (TPS) do not offer CT/CBCT dose calculation capability. Although the Monte Carlo algorithm can be used to simulate the radiation dose from $\mathrm{CT} / \mathrm{CBCT}$ scans, it is very time-consuming and usually requires Monte Carlo expertise. Hence, it would be highly desirable to have an easy-to-use tool that can be used to estimate the radiation dose from an imaging procedure performed on an individual anatomy.

\section{Development of an iphone app}

As the Apple iPhone is highly popular around the world, an iPhone app designed for such a purpose would be ideal to maximize its accessibility to the general public as well as medical practitioners. In October 2011, the CT Gently app project was initiated in collaboration with Komatsu Business Service Co. in Tokyo, Japan. The app was developed with Apple Xcode 4.2.1 toolkits with all the functionalities running on an Apple iPhone platform. The whole project was finished by June 2013 and the CT Gently app was released to the public for free downloads via Apple iTunes at https://itunes.apple.com/us/app/ ct-gently/id654734773?mt=8.

The philosophy for the interface design is two-folds: simplicity and efficiency. As shown in Figure 1, after the user inputs one's gender, age, weight, corresponding circumference at three anatomic sites (i.e., head, chest and abdomen) and given mAs and $\mathrm{kVp}$ values, CT Gently can generate comparative results both quantitatively and graphically on the organ doses and the associated cancer risks between the reference and the optimized settings for a CT or CBCT scan. It also allows the user to switch the units for weight and length between the international standard and the English system. In addition, a Question \& Answer (Q\&A) page can be accessed for simple explanations on the basic terms and concepts such as $\mathrm{CT}, \mathrm{CBCT}, \mathrm{mAs}, \mathrm{kVp}$, organ dose and relative risk etc.

\section{Organ dose estimation}

Based on Monte Carlo modeling of the CT and CBCT scans and particle transport simulation in human anatomy of various dimensions, the radiation doses to the various organs-at-risk (OARs) at the head, the chest and the abdomen regions can be obtained for a specific scan of given $\mathrm{mAs}$ and $\mathrm{kVp}$ values. ${ }^{5-12}$ In addition, personalized estimation of organ dose has been made possible based on some early cohort studies investigating the correlations between the organ dose and one's weight, circumference, scan mAs and $\mathrm{kVp} .^{9-12}$ Finally, to facilitate the calculation of organ dose, these correlations between the organ dose and the scan settings as well as anthropometric parameters have been formulated and implemented in CT Gently.

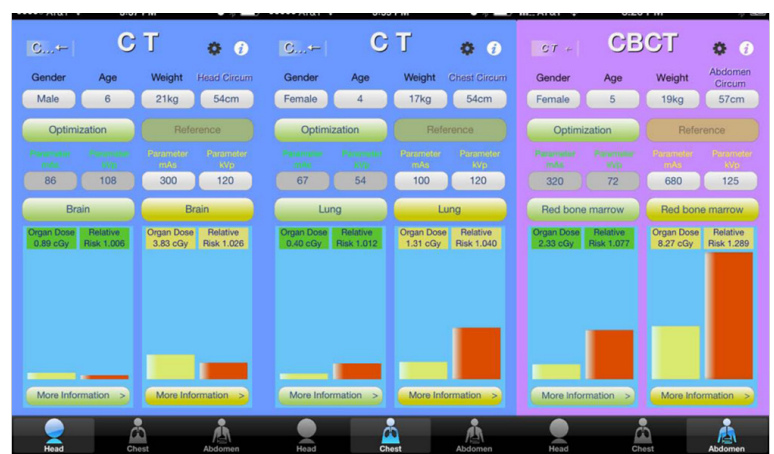

Figure I Screen shots of the CT Gently app running in the CT mode (left and middle panels) and CBCT mode (right panel), respectively. Specifically, a head CT scan is shown on the left with the mean radiation dose to the brain tissue and relative cancer risk calculated for the reference and optimized settings, respectively. Similarly, a chest CT scan and an abdominal CBCT scan are illustrated in the middle and right with lung tissue and red bone marrow as the critical structures, respectively. Screen shots of the CT Gently app running in the CT mode (left and middle panels) and CBCT mode (right panel), respectively. Specifically, a head $\mathrm{CT}$ scan is shown on the left with the mean radiation dose to the brain tissue and relative cancer risk calculated for the reference and optimized settings, respectively. Similarly, a chest CT scan and an abdominal $C B C T$ scan are illustrated in the middle and right with lung tissue and red bone marrow as the critical structures, respectively. 
Besides the reference settings of $\mathrm{mAs}$ and $\mathrm{kVp}$, a scan optimizer has been implemented to generate the optimized $m A s$ and $k V p$ settings for low-dose CT and CBCT scans based on the user's input. The optimizer works in such a way that almost constant low dose will be maintained for the specific organ-at-risk with uniform noise property, irrespective of one's physical dimension. In general, the optimized settings would reduce the doses to the OARs by $50-80 \%$ in comparison to the reference settings, which are the pre-defined settings from a scan protocol or a manufacturer, or a scan setting indicated by the user.

\section{Cancer risk estimation}

Using BEIR VII formulism, the radiation-induced excess relative risk (ERR) was calculated based on one's gender, age, and radiation dose to a certain OAR from a CT or CBCT scan. ${ }^{13}$ The relative risk (RR) is then reported as 1+ERR in the app. Qualitatively speaking, the relative risk stands for the excessive cancer risk related to the radiation exposure to a group of individuals, as compared to the group without radiation exposure.

\section{Discussion}

Designed to be simple and efficient, the CT gently app is intended not only for the clinicians but also for the general public. It is well known that scanner-specific features such as filtration design and inherent x-ray tube output play an important role in determining the organ dose. Confusion will arise among the general public if they are given too many technical choices such as scanner model, filtration and anode angle etc. Hence, limiting their choices to $\mathrm{mAs}$ and $\mathrm{kVp}$ besides their own anthropometric information would make this app simple and easy to use.

In addition, to develop the CT Gently app, we first applied Monte Carlo method to model the $\mathrm{kV}$ x-ray photon beams from a specific CT or CBCT scanner, and then used the developed model to simulate the dose distributions in real patient anatomy. Finally, based on retrospective cohort epidemiologic studies, we obtained the correlations between the patient size, $\mathrm{mAs}, \mathrm{kVp}$ and the organ dose, and implemented them in the app. As there are numerous $\mathrm{CT}$ and $\mathrm{CBCT}$ scanner models available in the market, it would be practically impossible to go for this route of Monte Carlo modeling and simulation on a large number of patients. Hence, it is important to realize that the results reported in this app are scanner-dependent. That is, for CT scans, the results are based on a Siemens Somatom Sensation 16 scanner while a Varian On-board Imager system has been used as a base model for CBCT scans. If the user's model is different from the base model, a normalization factor (CTDI ${ }_{\text {vol, base' }}$ $\mathrm{CTDI}_{\text {vol user }}$ ) will be required to convert the organ dose reported for the base model in the app to the user's model.

\section{Conclusion}

An iPhone app has been developed as a mobile tool to estimate the organ doses and the associated cancer risks from CT and CBCT scans based on individual anatomy and scan mode. Furthermore, this easy-to-use app can be used to generate optimized settings for personalized low-dose $\mathrm{CT}$ and $\mathrm{CBCT}$ scans. With the increasing concern of radiation dose and cancer risk among the clinicians and the general public, CT Gently may help increase the awareness about the safe and appropriate applications of medical imaging in the clinic with improved benefit-to-risk ratio in the long run, in accordance with the 'Image Gently' and 'Image Wisely' campaigns..$^{14,15}$

\section{Future developments}

Currently, CT Gently only reports organ dose and cancer risk for one organ per scan site. That is, the brain for head scans, the lung for chest scans, and the red bone marrow for abdominal scans. More organs will be included in the future release to expand this functionality. In addition, CT Gently currently generates one set of optimized settings based on one's circumference, which is basically a balanced approach to reducing the organ dose while maintaining acceptable image quality. In the future, two more optimization scenarios may be added to suit different clinical needs, i.e., to better spare the organs or to achieve better image quality. Finally, the Android and Windows-based versions of CT Gently are also expected in the near future.

\section{Acknowledgments}

None.

\section{Conflicts of interest}

Authors declare there are no conflicts of interest.

\section{References}

1. Brenner DJ, Hall EJ. Computed tomography--an increasing source of radiation exposure. $N$ Engl J Med. 2007;357(22):2277-2284.

2. Dawson LA, Sharpe MB. Image-guided radiotherapy: rationale, benefits, and limitations. Lancet Oncol. 2006;7(10):848-858.

3. Pearce MS, Salotti JA, Little MP, et al. Radiation exposure from CT scans in childhood and subsequent risk of leukaemia and brain tumours: a retrospective cohort study. Lancet. 2012;380(9840):499-505.

4. Brenner DJ. Is it time to retire the CTDI for CT quality assurance and dose optimization? Med Phys. 2005;32(10):3225-3226.

5. Angel E, Yaghmai N, Jude CM, et al. Dose to radiosensitive organs during routine chest CT: effects of tube current modulation. AJR Am J Roentgenol. 2009;193(5):1340-1345.

6. Reid J, Gamberoni J, Dong F, et al. Optimization of $\mathrm{kVp}$ and $\mathrm{mAs}$ for pediatric low-dose simulated abdominal CT: is it best to base parameter selection on object circumference? AJR Am J Roentgenol. 2010;195(4):1015-1020.

7. Zhang Y, Li X, Segars WP, et al. Organ doses, effective doses, and risk indices in adult CT: comparison of four types of reference phantoms across different examination protocols. Med Phys. 2012;39(6):3404-3423.

8. Lee C, Kim KP, Long DJ, et al. Organ doses for reference pediatric and adolescent patients undergoing computed tomography estimated by Monte Carlo simulation. Med Phys. 2012;39(4):2129-2146

9. Zhang Y, Yan Y, Nath R, et al. Personalized estimation of dose to red bone marrow and the associated leukaemia risk attributable to pelvic kilo-voltage cone beam computed tomography scans in image-guided radiotherapy. Phys Med Biol. 2012;57(14):4599-4612.

10. Zhang $\mathrm{Y}$, Yan $\mathrm{Y}$, Nath R, et al. Personalized assessment of $\mathrm{kV}$ cone beam computed tomography doses in image-guided radiotherapy of pediatric cancer patients. Int J Radiat Oncol Biol Phys. 2012;83(5):1649-1654.

11. Deng J, Chen Z, Yu JB, et al. Testicular doses in image-guided radiotherapy of prostate cancer. Int $J$ Radiat Oncol Biol Phys. 2012;82(1):e39-e47.

12. Deng J, Chen Z, Roberts KB, et al. Kilovoltage imaging doses in the radiotherapy of pediatric cancer patients. Int J Radiat Oncol Biol Phys. 2012;82(5):1680-1688.

13. National Research Council. Health risks from exposure to low levels of ionizing radiation: BEIR VII - Phase 2. Committee to Assess Health Risks from Exposure to Low Levels of Ionizing Radiation. National Academies Press: Washington, DC, USA; 2006. 
14. Goske MJ, Applegate KE, Boylan J, et al. The 'Image Gently' campaign: increasing CT radiation dose awareness through a national education and awareness program. Pediatr Radiol. 2008;38(3):265-269.
15. Brink JA, Amis ES Jr. Image Wisely: a campaign to increase awareness about adult radiation protection. Radiology. 2010;257(3):601-602. 